Article

\title{
Design of an Electrically Tunable Micro-Lens Based on Graded Photonic Crystal
}

\author{
YongLe $\mathrm{Qi}^{1}{ }^{1}{ }^{(\mathbb{C}}$, XiaoHong Sun ${ }^{1, *}$, Shuai Wang ${ }^{1}{ }^{(\mathbb{D})}$, Wen Yang $\mathrm{Li}^{2}$ and ZhongYong Wang ${ }^{1}$ \\ 1 Henan Key Laboratory of Laser and Opto-Electric Information Technology, School of Information \\ Engineering, Zhengzhou University, Zhengzhou 450066, China; optylqi@gmail.com (Y.L.Q.); \\ zzuwangs@gmail.com (S.W.); iezywang@zzu.edu.cn (Z.Y.W.) \\ 2 Wuhan National Laboratory for Optoelectronics, Huazhong University of Science and Technology, \\ Wuhan 430074, China; D201677619@hust.edu.cn \\ * Correspondence: iexhsun@zzu.edu.cn; Tel.: +86-137-2142-3613
}

Received: 25 May 2018; Accepted: 19 July 2018; Published: 23 July 2018

\begin{abstract}
A micro-lens with an adjustable focal length (FL) is designed by using Graded Photonic Crystal (GPC) structures and a Polymer Dispersed Liquid Crystal (PDLC) material. The GPCs are formed by gradually changing the radius of the polymer rods in the Photonic Crystal (PC) with square lattices of polymer rods in the background of Liquid Crystals (LCs). The electrically tunable focusing characteristics of the micro-lens are investigated by loading a continuous voltage source to change the LC rotation angle. The sensitivity of the focal shift in terms of LCs tilting angle is $0.152 \lambda(\mathrm{nm} / \mathrm{deg})$. Moreover, the effect of the defects and deviations on the focusing characteristics are also analyzed. This research is crucial for future applications of the proposed device in the integrated photonics and adaptive optics.
\end{abstract}

Keywords: Graded Photonic Crystals; Polymer Dispersed Liquid Crystal; focal length; adjustable

\section{Introduction}

The optical lens is an indispensable component for various optical setups and systems such as cameras, projectors and imagers. With advances in various technologies such as smartphones, virtual and augmented reality, and healthcare instruments, high performance lenses are in high demand. As pointed out by $\mathrm{H}$ Ren and $\mathrm{ST} \mathrm{Wu}$, adaptive lenses have a broad prospect in the future applications [1]. Different approaches have been explored to achieve tunable lenses. For example, meniscuses between water and oil were used as an optical lens; its focal length can be adjusted by varying the surface curvature [2]. Polymer micro-lenses can be tuned by using heat [3]. One fundamental challenge is that the spherical surface profiles of these lenses are inevitably accompanied by spherical aberrations. Lenses using liquid crystals (LCs) have been extensively utilized for beam steering and making lenses with adjustable liquid crystal lenses. LCs are great candidates for tunable lenses owing to their optical anisotropy and electrically tunable orientation. Numerous LC lens and micro-lens arrays are reported for 3D imaging and sensing system construction [4], electrically switchable 2D/3D modes [5], ophthalmic applications [6], pico-projectors [7], and optical zoom systems [8]. However, the lens manufacturing processes mentioned in these reports are complicated and are not conducive to large-scale manufacturing.

Graded Photonic Crystals (GPCs) consist of unit cells with spatially varying geometrical or material properties [9]. GPCs with engineered spatial gradients can be used in devices for focusing and guiding light [10-14]. The emerging fields of metasurfaces utilize nanoscale resonators or wave guides to fine tune the amplitude, phase and polarization of the optical fields, making possible various applications such as lensing, holography and beam shaping [15-20]. Different methods have been 
employed in order to perform auto-focusing of light including electro-optical [2], thermo-optical [3], electro-mechanical [21] and acousto-mechanical [22] techniques. Among these techniques, LCs are considered to be good choices for electro-optical adaptive lens design since applying a control voltage gives rise to a dynamical tuning in the orientations of the LCs molecules, and consequently, the effective refractive index of the LCs changes based on the voltage levels [23]. LCs may also have great potential in adaptive optics implementations due to their simple electrooptical control, low control voltage, compactness, low power dissipation, and relatively low cost [24]. Combining LCs and GPCs can have both the advantage of precise wave front engineering and large tunability.

A micro-lens with adjustable focal length (FL) is designed, which is composed of GPCs and LCs. This design can be made quickly by a single-step interference-based approach [25]. The GPCs are kinds of square lattices with polymer rods in the background of LCs. The flexible polymer has a refractive index of $n=1.76$ in the visible spectrum. The electrically tunable micro-lens not only has adjustable FL, but also has high fault tolerance.

\section{Design of GPC Micro-Lens of Polymer Rods in the Air Background}

\subsection{Basic Structure}

A PC structure with square lattices is considered and composed of polymer rods and air background. The GPC structure is formed by changing the rods size. The radius of the rods can be designed by the effective medium theory [26]. The unit cell of PC is shown in Figure 1a, where the rod is a polymer and the background are PCs. According to the Maxwell-Garnett effective medium theory, the equivalent dielectric constant of different polarization states can be given by the following formula [26],

$$
\begin{aligned}
& \varepsilon_{\text {eff,TE }}=\varepsilon_{b} \frac{(1-\mathrm{F}) \varepsilon_{b}+(1+\mathrm{F}) \varepsilon_{\text {rod }}}{(1+\mathrm{F}) \varepsilon_{b}+(1-\mathrm{F}) \varepsilon_{\text {rod }}} \\
& \varepsilon_{\text {eff,TM }}=(1-\mathrm{F}) \varepsilon_{b}+\mathrm{F} \times \varepsilon_{\text {rod }}
\end{aligned}
$$

where $\varepsilon_{b}$ is the dielectric constant of the background, $\varepsilon_{r o d}$ is the dielectric constant of dielectric rod, $\mathrm{F}$ is the filling fraction of the polymer rods, i.e., $\mathrm{F}=\pi \mathrm{r}^{2}$ rod $/ \mathrm{a}^{2}$, where a is lattice constant. Therefore, the radius of the rods can be expressed as [9],

$$
\begin{aligned}
& \mathrm{r}_{\text {rod,TE }}=\mathrm{a} \times \sqrt{\frac{\left(\varepsilon_{\text {rod }}+\varepsilon_{b}\right)\left(\varepsilon_{e f f}-\varepsilon_{b}\right)}{\pi\left(\varepsilon_{\text {rod }}-\varepsilon_{b}\right)\left(\varepsilon_{e f f}+\varepsilon_{b}\right)}} \\
& \mathrm{r}_{\text {rod,TM }}=\mathrm{a} \times \sqrt{\frac{\left(\varepsilon_{e f f}-\varepsilon_{b}\right)}{\pi\left(\varepsilon_{e f f}-\varepsilon_{b}\right)}}
\end{aligned}
$$

TM lens can lead to a larger transmission bandwidth and better focusing characteristics compared with those of TE lens [27]. In this work, we mainly study the issue of TM polarization. A row of the GPCs is shown in Figure 1c. The effective index distribution of the GPC design is expressed as the equation

$$
\mathrm{n}_{\text {eff }}(\mathrm{y})=\mathrm{n}_{0}\left[1-\frac{1}{2}(\text { Ay })^{2}\right]
$$

in which $A=2 \pi / 8 r_{\text {rod }}$. The designed GPCs has an effective index distribution varying from $n_{\text {eff }}$ $(y= \pm 10 a)=1.22$ to $n_{\text {eff }}(y=0)=1.62$. The corresponding effective index and the radii of the rod distribution are depicted in Figure 1. Considering Equations (1) and (2) and the effective refractive index distribution Equation (3), the radii of the GPC polymer rods can be calculated from $r_{\text {rod }}$ $(y= \pm 10 a)=0.1968$ a to $r_{\text {rod }}(y=0)=0.4964 a$. 
(a)

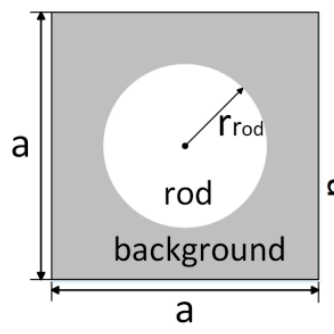

(b)

(c)

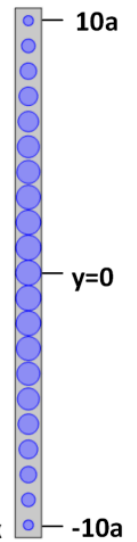

Figure 1. (a) The unit cell consists of rod and background; (b) Curves of effective refractive index and dielectric rod radius varying with $\mathrm{Y}$ direction (c) Schematic diagram of single layer structure.

\subsection{Model Creation}

The structure schematic diagram of the GPCs is shown in Figure 2a-c. The GPCs are composed of 80 columns of the single layer structure. The main parameters of the structure include the effective refractive index distribution, the radii of the GPC polymer rods (were calculated in Section 2.1) and the lattice constant $\mathrm{a}=150 \mathrm{~nm}$. The incident light is parallel with the surface of the GPCs, as the color arrow shown in Figure $2 b$. The 3D schematic diagram of TM light irradiation to the micro-lens is shown in Figure 2a. The $x$-axis, is the direction of light propagation, and the green arrow expresses the direction of magnetic field vibration. There is no magnetic field component in the direction of the incident propagation. The focusing properties of GPCs can be obtained by observing the electric field distribution.

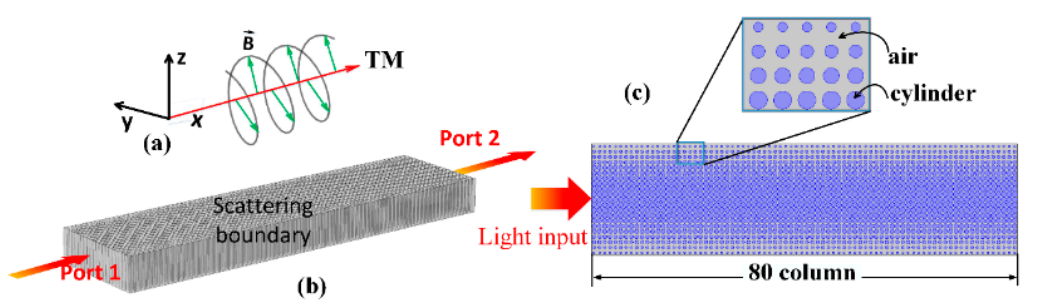

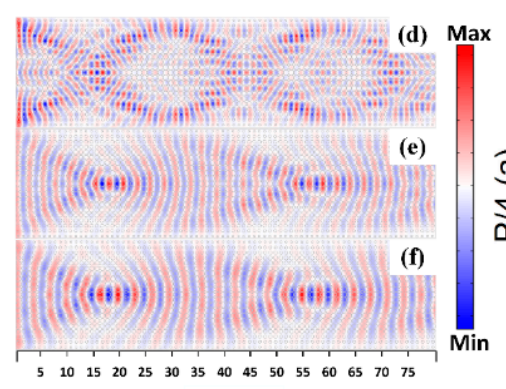

Length(a)

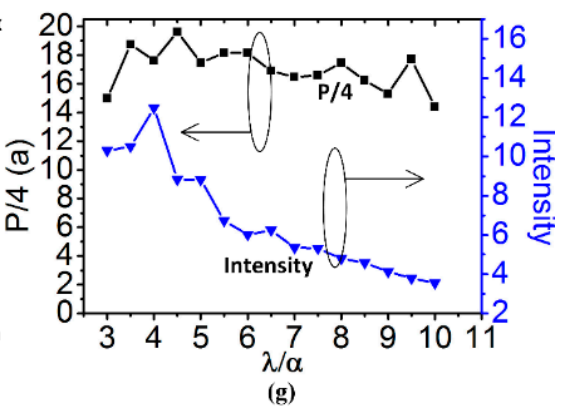

Figure 2. (a) The direction of the magnetic field vibration in the light wave (b) 3D structure model of the GPCs (c) 2D structure model of the GPCs. The electric field distributions at (d) $\lambda / a=3$ (e) $\lambda / a=4$ and (f) $\lambda / \mathrm{a}=5(\mathrm{~g})$. The $\mathrm{P} / 4$ and the relative intensity of light at focal point variation with the various of the incident wavelength.

The finite element method (FEM) is used for the simulation. As shown in Figure 2, the left and right boundaries are set as port 1 and port 2, where port 1 is the excitation source with TM mode. 
The other boundaries are set to scattering boundary. The refractive indices of air and rods are set as 1 and 1.76, respectively. The whole model is divided by free tetrahedron with a specially refined mesh.

\subsection{Focusing Characteristics}

The electric field distributions of light from $450 \mathrm{~nm}$ to $1550 \mathrm{~nm}$ in the GPCs are calculated. All the electric field distributions are selected from the top surface of the GPC structure. The distance between the two focusing points in the structure is half-pitch $(\mathrm{P} / 2)$. The light goes from the left side and is focused on the $1 / 4$ pitch $(\mathrm{P} / 4)$. The value of $\mathrm{P} / 4$ changes from in the range of $14.4 \mathrm{a}$ to $19.6 \mathrm{a}$, as shown in Figure $3 \mathrm{~g}$. The intensity of the focusing point reaches the maximum at $\lambda / \mathrm{a}=4$. Figure $2 \mathrm{~d}-\mathrm{f}$ show the electric field distributions at $\lambda / a=3,4$ and 5 , in which the half pitch between two focusing points are different and they are $30.00 a, 35.24 a$ and $34.94 a$, respectively.

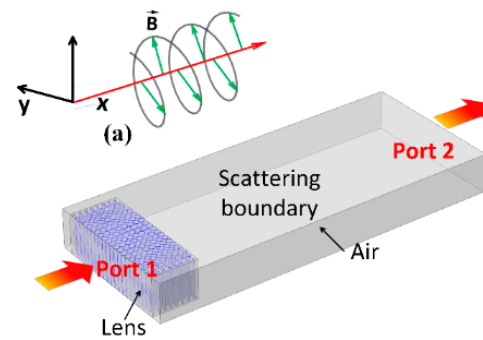

(b)

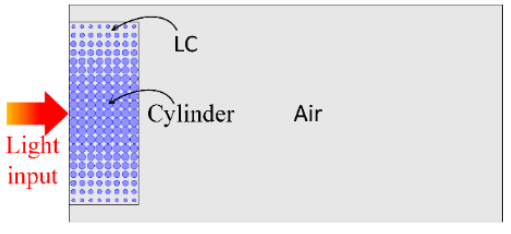

(c)

Figure 3. (a) The direction of the magnetic field vibration in the light wave. (b) 3D structure model (c) 2D structure model.

It can be predicted that if the thickness of the GPC lens is $1 / 4$ pitch, the FL is 0 . If there is only a single layer, the light will be focused at a long distance. The thinner the lens, the longer the FL.

\section{Electrically Tunable Characteristics of PDLC Based GPC Micro-Lens}

\subsection{Principle}

Based on the above GPC structure, we can obtain the PDLC micro-lens by replacing the air background with the LCs. The FL of the PDLC lens can be adjusted by changing the voltage, as the LC has electronic controlled birefringence effect. For TM polarization, the effective index of LCs can be expressed by the following equation [28]:

$$
\mathrm{n}_{\mathrm{LC}}^{2}=\frac{\mathrm{n}_{\mathrm{e}}^{2} \mathrm{n}_{\mathrm{o}}^{2}}{\mathrm{n}_{\mathrm{e}}^{2} \cos ^{2}(\theta)+\mathrm{n}_{\mathrm{o}}^{2} \sin ^{2}(\theta)}
$$

where $\theta$ is the rotation angle. The effective index of LCs molecules varies from $n_{L C}=n 0$ at $\theta=0^{\circ}$ to $\mathrm{n}_{\mathrm{LC}}=\mathrm{n}_{\mathrm{e}}$ at $\theta=90^{\circ}$. The rotation angle of LCs can be tuned with an external electric field exerted on Indium tin oxide (ITO) layers. The relationship between the tilting angle and the voltage can be expressed as

$$
\theta=\frac{\pi}{2}-2 \tan ^{-1} \exp \left(-\mathrm{V}_{\mathrm{RMS}}\right),\left(\mathrm{V}_{\mathrm{RMS}}>0\right)
$$

where $V_{\mathrm{RMS}}$ is the normalized root mean square (RMS) voltage.

\subsection{Electrically Tunable Micro-Lens}

To operate as a focusing lens, the transverse and longitudinal dimensions of the designed GPCs are chosen as $8 \mathrm{a}$ and 21a, respectively. As an example, $\lambda=600 \mathrm{~nm}$ and $\mathrm{a}=150 \mathrm{~nm}$ are selected for analysis. The simulation model is built in the same way as Section 2. The polarization diagram of incident light, the $3 \mathrm{~d}$ and $2 \mathrm{~d}$ diagrams of the simulation model are shown in Figure $3 \mathrm{a}-\mathrm{c}$, respectively. The simulation model consists of the rods, LCs and air. Their refractive indices of rods and air are 1.76 and 1. The refractive index of LCs is related to voltage, and its range is from 1.59 to 2.2. 
As shown in Figure 4a, ITO glass is chosen as electrodes to change the voltage and adjust the LC refractive index. The effective index distribution of the designed lens is plotted in Figure $4 \mathrm{~b}$. The background is air or LCs with different rotation angles of $\theta=0^{\circ}, 38^{\circ}, 90^{\circ}$. To better observe the lens focusing tunability, the steady state electric field intensities for the above three angles are calculated and shown in Figure 4c,e, respectively. A strong focusing effect is observed in the case of $\theta=0^{\circ}$ (in the Figure 4c) whereas the incident beam is not affected much inside the GPC medium when the rotation angle is set to $\theta=38^{\circ}$ (Figure 4d). The spatial electric field distribution in Figure $4 \mathrm{~d}$ resembles a propagating beam that encounters a pure dielectric slab. While setting the rotation angle to $\theta=90^{\circ}$, the proposed configuration starts to operate as a diverging lens and thus, the propagating beam diverges inside the structure, as shown in Figure 4e. The simulation results imply that the focusing characteristic of the PDLC lens can be controlled by the LC orientation or voltage.

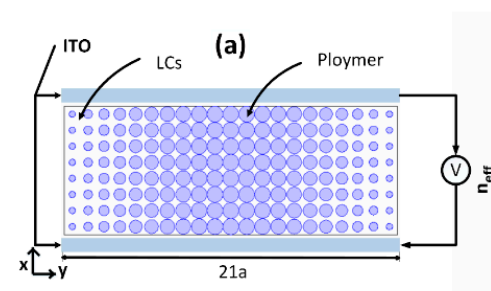

(b)

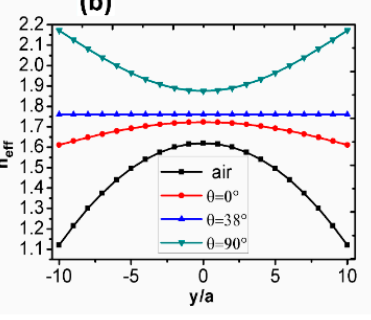

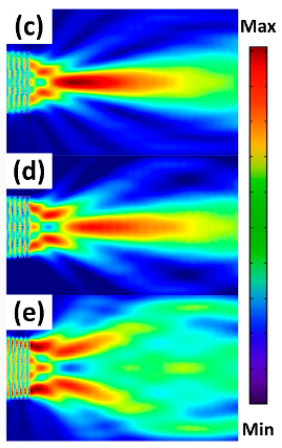

Figure 4. (a) The schematic of electrically tunable micro-lens. (b) Effective refractive index distribution with different background of air or LCs with the various of the rotation angles. The electric field intensities of micro-lens with rotation angle of $(\mathbf{c}) \theta=0^{\circ}$ (d) $\theta=38^{\circ}$ (e) $\theta=90^{\circ}$.

The electrical tunable characteristics of the lens are investigated by assuming loading a continuous voltage source to the two sides of the lens. The FL as well as the full width at half maximum (FWHM) values of the proposed lens are calculated with different $\theta$ and plotted in Figure 5a. The FL increases from 14.365a (when $\theta=5^{\circ}$ ) to 19.067a (when $\theta=35^{\circ}$ ). In this range, the curve of FL can be fitted as a primary function, $\mathrm{FL}(\theta)=\mathrm{m}+\mathrm{n} \theta$, where $\mathrm{m}, \mathrm{n}$ are constant parameters given by $\mathrm{m}=14.23 \mathrm{a}$, $\mathrm{n}=0.15 \mathrm{a}$. Meanwhile, the FWHM of the lens system increases from $1.21 \lambda$ to $1.44 \lambda$. Compared with the size of the entire lens, the enlargement of FWHM is relatively small. The effective refractive index distribution in the cases of $\theta=\left\{10^{\circ}, 20^{\circ}, 30^{\circ}\right\}$ is shown in Figure $5 \mathrm{~b}$. In Figure $5 \mathrm{c}, \mathrm{e}$, the electric field intensity profiles at the three different rotation angles are represented to observe the focal point shift as well as the focusing power variations with respect to rotation angle changes.

(a)

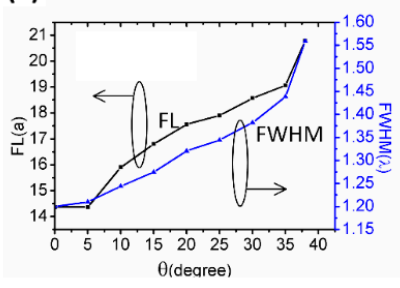

(b)

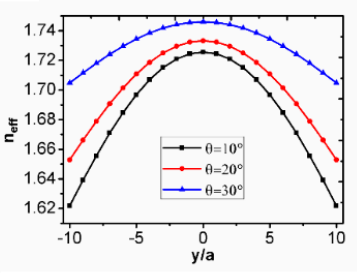

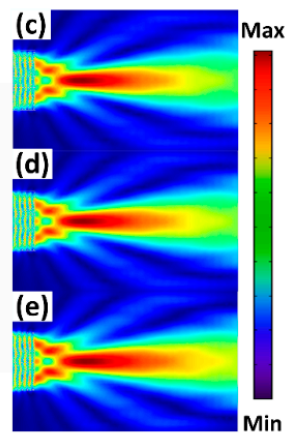

Figure 5. (a) The FL and FWHM changes with rotation angles; (b) Effective refractive index profile of GPC lens for LC with different rotation angles. The electric field intensities distributions of micro-lens with rotation angle at $(\mathbf{c}) \theta=10^{\circ}$ (d) $\theta=20^{\circ}$ (e) $\theta=30^{\circ}$. 
The sensitivity of the focal shift in terms of LCs tilting angle is $\Delta \mathrm{FL} / \Delta \theta=0.152 \lambda(\mathrm{nm} / \mathrm{deg})$, which proves that vision correction and auto-focusing applications can be provided with such PDLC lens design. The FL varies linearly in a certain range of voltage, which provides a great convenience in the use of the lens.

\section{The Effect of the Lens Defects and Deviations on Focusing Characteristics}

In the process of making the lens, some defects cannot be avoided. In order to evaluate this effect of defects on the performance of the lens, the effect of the dielectric rods structure with defects and deviations are tested in this section. In order to facilitate data comparison, the values of $\lambda$ and a are consistent with those in Section 3.

Three different cases of defects: (1) lacking of a row of rods on the edge, (2) lacking of a corner at the front and (3) lacking of a corner at the back. The schematic diagrams of defects in different cases are shown in Figure 6a,c. The corresponding FWHM and FL variations based on the normalized RMS voltage are shown in Figure 6d,e, respectively. As shown in these figure, a small defect has little effect on the performance of the lens.
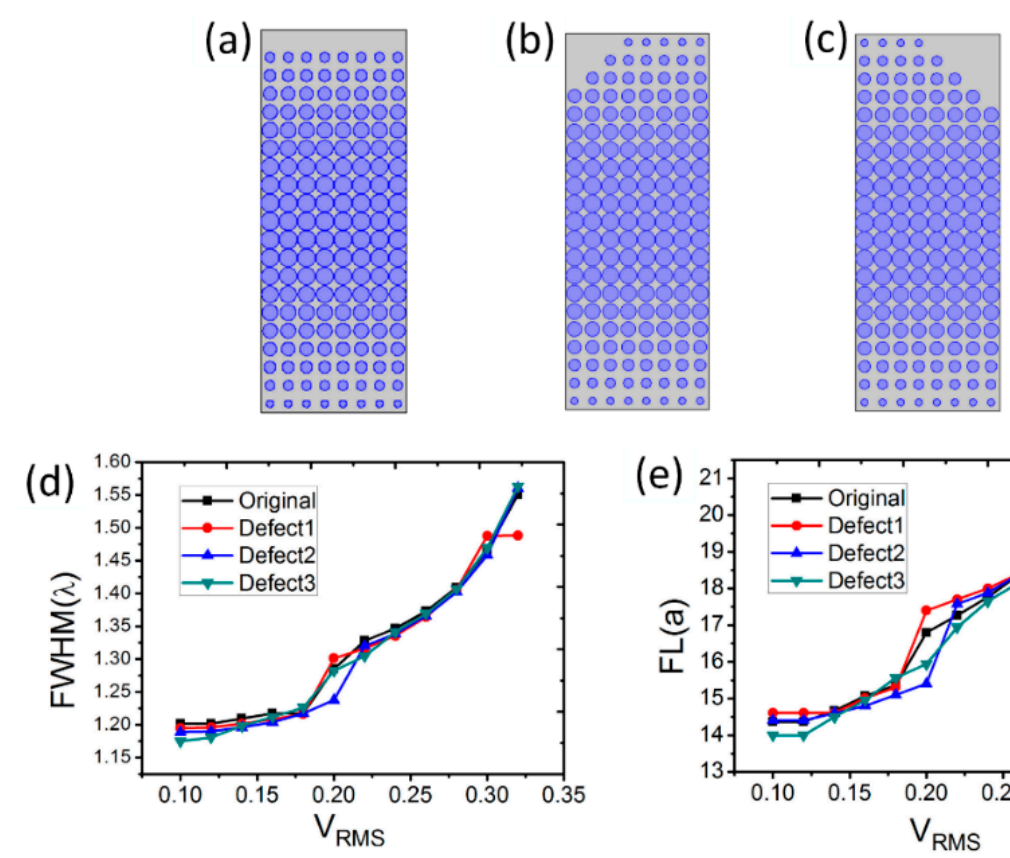

Figure 6. Schematic diagram of defects for (a) lack of a row of rod on the edge (b) lacking of a corner at the front (c) lacking of a corner at the back; (d) The FWHM and (e) the FL curves with the RMS voltage variation under different defects.

The structural diagrams of $-6 \%,-8 \%$ and $-10 \%$ radii deviations are shown in Figure $7 \mathrm{a}, \mathrm{c}$. The curves of the FWHM changing with normalized RMS voltage are shown in Figure 7d. With the low voltage $(<0.22)$, the FWHM widens with the increase of the radii deviation. When $\mathrm{V}_{\mathrm{RMS}}>0.22$, the radii deviation within 10\% has a little impact on the FWHM. Figure 7e shows that the curve of the FL varies with the normalized RMS voltage. It can be seen clearly that the FL curve have an obvious differentiation of the different radii deviation greater than $8 \%$ and less than $6 \%$. The FL curves of $6 \%$ radii deviation and the original almost coincide, i.e., the deviation within $6 \%$ does not affect the result. 
(a)

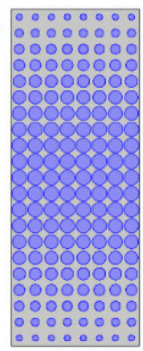

(b)

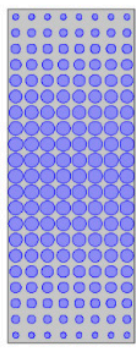

(c)

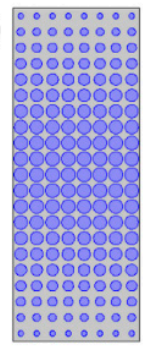

(d)

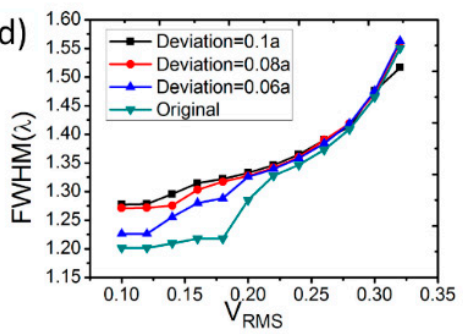

(e)

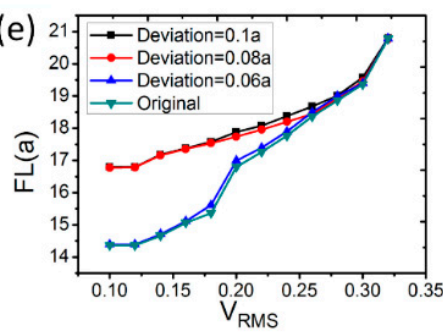

Figure 7. Schematic diagram of a cylindrical radii deviation of (a) $-6 \%$ (b) $-8 \%$ (c) $-10 \%$. (d) The FWHM and (e) the FL curves with the RMS voltage variation under different deviations.

Therefore, it can be claimed that the studied PDLC lens system is robust to a small amount of defects and deviation within $6 \%$ that may arise during fabrication.

\section{Conclusions}

The PDLC-based electrically tunable micro-lens has been designed by using a GPC structure. The FL of the lens has a wide adjustable range and can be accurately controlled. The calculated results show that the FL in the visible spectrum can be tuned from 14.365a to 19.067a when the LC rotation angles change from $5^{\circ}$ to $35^{\circ}$. The corresponding focal shift is $\Delta \mathrm{FL} / \Delta \theta=0.152 \lambda(\mathrm{nm} / \mathrm{deg})$. The slight edge defects and $6 \%$ radius deviations have little effect on the focusing characteristics of the lens. This investigation will provide theoretical guidance for developing the devices of near-field imaging, auto-focusing, integrated optical circuit and optical communication.

Author Contributions: Data curation, Y.L.Q.; Funding acquisition, X.H.S. and Z.Y.W.; Investigation, W.Y.L.; Methodology, Y.L.Q. and X.H.S.; Resources, X.H.S.; Software, S.W.; Validation, Y.L.Q.; Writing-original draft, Y.L.Q.; Writing—review \& editing, X.H.S.

Funding: This research was funded by Science and Technology Major Project of Henan Province (161100210200) and Opening Foundation (HZ2016-KF01) of State Key Laboratory of Fire Science, University of Science and Technology of China.

Conflicts of Interest: The authors declare no conflict of interest.

\section{References}

1. Ren, H.; Wu, S.T. Introduction to Adaptive Lenses; Wiley Series in Pure and Applied Optics; Wiley: Hoboken, NJ, USA, 2012.

2. Dong, L.; Agarwal, A.K.; Beebe, D.J.; Jiang, H. Adaptive liquid microlenses activated by stimuli-responsive hydrogels. Nature 2006, 442, 551. [CrossRef] [PubMed]

3. Huang, X.; Cheng, C.M.; Wang, L.; Wang, B.; Su, C.C.; Ho, M.S.; LeDuc, P.R.; Lin, Q. Thermally tunable polymer microlenses. Appl. Phys. Lett. 2008, 92, 251904. [CrossRef]

4. Hassanfiroozi, A.; Huang, Y.P.; Javidi, B.; Shieh, H.P.D. Hexagonal liquid crystal lens array for 3D endoscopy. Opt. Express 2015, 23, 971. [CrossRef] [PubMed]

5. Huang, Y.P.; Jen, T.H.; Chang, Y.C.; Shieh, P.Y.; Chen, C.W.; Liao, L.Y. Individually adapted LC-lens array for 3D applications. Mol. Cryst. Liquid Cryst. 2014, 605, 267-274. [CrossRef] 
6. Lin, Y.H.; Chen, H.S. Electrically tunable-focusing and polarizer-free liquid crystal lenses for ophthalmic applications. Opt. Express 2013, 21, 9428. [CrossRef] [PubMed]

7. Lin, H.C.; Lin, Y.H. An electrically tunable focusing pico-projector adopting a liquid crystal lens. Jpn. J. Appl. Phys. 2010, 49, 102502. [CrossRef]

8. Lin, Y.H.; Chen, M.S.; Lin, H.C. Electrically-tunable optical zoom system by using liquid crystal lenses. In Proceedings of the Advances in Display Technologies II, International Society for Optics and Photonics, San Francisco, CA, USA, 25-26 January 2012.

9. Vasić, B.; Gajić, R. Self-focusing media using graded photonic crystals: Focusing, Fourier transforming and imaging, directive emission, and directional cloaking. J. Appl. Phys. 2011, 110, 053103. [CrossRef]

10. Chien, H.T.; Chen, C.C. Focusing of electromagnetic waves by periodic arrays of air holes with gradually varying radii. Opt. Express 2006, 14, 10759. [CrossRef] [PubMed]

11. Roux, F.S.; Leon, I.D. Planar photonic crystal gradient index lens, simulated with a finite difference time domain method. Phys. Rev. B 2006, 74, 113103. [CrossRef]

12. Kurt, H.; Colak, E.; Cakmak, O.; Caglayan, H.; Ozbay, E. The focusing effect of graded index photonic crystals. Appl. Phys. Lett. 2008, 93, 171108. [CrossRef]

13. Wu, Q.; Gibbons, J.M.; Park, W. Graded negative index lens by photonic crystals. Opt. Express 2008, 16, 16941-16949. [CrossRef] [PubMed]

14. Falek, E.; Shavit, R. 2D flat lens design made of dielectric cylinders using the array scattering method. In Proceedings of the IEEE International Symposium on Antennas and Propagation \& USNC/URSI National Radio Science Meeting, San Diego, CA, USA, 9-14 July 2017; pp. 1257-1258.

15. Scheuer, J. Metasurfaces-based holography and beam shaping: Engineering the phase profile of light. Nanophotonics 2016, 6, 137-152. [CrossRef]

16. Ye, X.; Qi, L. Two-dimensionally patterned nanostructures based on monolayer colloidal crystals: Controllable fabrication, assembly, and applications. Nano Today 2011, 6, 608-631. [CrossRef]

17. Chen, F. Micro- and submicrometric waveguiding structures in optical crystals produced by ion beams for photonic applications. Laser Photonics Rev. 2012, 6, 622-640. [CrossRef]

18. Shen, Y.; Hsu, C.W.; Yeng, Y.X.; Joannopoulos, J.D.; Soljačić, M. Broadband angular selectivity of light at the nanoscale: Progress, applications, and outlook. Appl. Phys. Rev. 2016, 3, 011103. [CrossRef]

19. Liu, Z.W.; Wei, Q.H.; Zhang, X. Surface plasmon interference nanolithography. Nano Lett. 2005, 5, 957-961. [CrossRef] [PubMed]

20. Lin, H.C.; Chen, M.S.; Lin, Y.H. A Review of Electrically Tunable Focusing Liquid Crystal Lenses. Trans. Electr. Electron. Mater. 2011, 12, 231-236. [CrossRef]

21. Wang, H.W.; Chang, I.L.; Chen, L.W. Beam manipulating by graded photonic crystal slab made of dielectric elastomer actuators. Opt. Commun. 2012, 285, 5524-5530. [CrossRef]

22. Olles, J.D.; Vogel, M.J.; Malouin, B.A.; Hirsa, A.H. Optical performance of an oscillating, pinned-contact double droplet liquid lens. Opt. Express 2011, 19, 19399-19406. [CrossRef] [PubMed]

23. Jewell, S. Polarized Light in Liquid Crystals and Polymers. Liquid Cryst. Today 2009, 18, 59-60. [CrossRef]

24. Purvis, A. A real-time closed-loop liquid crystal adaptive optics system: First results. Opt. Commun. 1997, 137, 17-21.

25. Behera, S.; Joseph, J. Single-step optical realization of bio-inspired dual-periodic motheye and gradient-index-array photonic structures. Opt. Lett. 2016, 41, 3579-3582. [CrossRef] [PubMed]

26. Datta, S.; Chan, C.T.; Ho, K.M.; Soukoulis, C.M. Effective dielectric constant of periodic composite structures. Phys. Rev. B 1993, 48, 14936. [CrossRef]

27. Sun, X.H.; Wu, Y.L.; Liu, W.; Hao, Y.; Jiang, L.D. Luneburg lens composed of sunflower-type graded photonic Crystals. Opt. Commun. 2014, 315, 367-373. [CrossRef]

28. Prost, J. The Physics of Liquid Crystals; Oxford University Press: London, UK, 1995.

(C) 2018 by the authors. Licensee MDPI, Basel, Switzerland. This article is an open access article distributed under the terms and conditions of the Creative Commons Attribution (CC BY) license (http:/ / creativecommons.org/licenses/by/4.0/). 